\title{
Positron Annihilation Study of Defects in the Cage-Type Uranium Compound
}

\author{
A. RubaszeK*, \\ W. Trzebiatowski Institute of Low Temperatures and Structure Research, Polish Academy of Sciences \\ P.O. Box 1410, 50-950 Wrocław 2, Poland
}

\begin{abstract}
The electron and positron properties of the $\mathrm{UIr}_{2} \mathrm{Zn}_{20}$ compound of the $F d-3 m$ cage-type crystal structure are studied. The characteristics for the perfect crystal are compared with their counterparts in the elements containing Ir and $\mathrm{Zn}$ vacancy. The presence of the vacancy hardly influences the electron charge density around individual atoms. In contrast to electrons, the positron is strongly localized inside the vacancy and the transfer of positron charge is observed. The positron lifetime, $\tau$, appears to be strongly sensitive to the presence and type of the vacancy. Furthermore, this parameter calculated for the perfect crystal is very close to the experimental value for $\mathrm{Zn}$. Here the theoretical values of $\tau$ depend on the approach applied to the electron positron (e-p) correlations.
\end{abstract}

DOI: 10.12693/APhysPolA.125.737

PACS: $78.70 . \mathrm{Bj}$

\section{Introduction}

The increasing interest in the uranium based intermetallic compounds of type $\mathrm{UT}_{2} \mathrm{Zn}_{20}$, where $\mathrm{T}$ is a transition metal (cf. [1,2] and references cited therein), is due to outstanding physical and structural properties of these materials. $\mathrm{UT}_{2} \mathrm{Zn}_{20}$ crystallise in the specific cage-type $F d-3 m$ cubic structure. The $\mathrm{U}$ atoms are separated inside the polyhedrons formed by $16 \mathrm{Zn}$ atoms with closed $d$ shells and the $\mathrm{U}-\mathrm{U}$ inter-atomic distances exceed 12 atomic units (a.u.). In consequence, the $f$ electrons' hybridisation with ligands is very weak and the magnetic exchange hardly occurs.

In particular, the basic physical properties of the $\mathrm{UIr}_{2} \mathrm{Zn}_{20}$ crystals, that have been recently measured by various research groups [1, 2], exhibit essential discrepancies. In Ref. [2] the compound $\mathrm{UIr}_{2} \mathrm{Zn}_{20}$ was classified as an itinerant heavy fermion that orders ferromagnetically at $2.1 \mathrm{~K}$, in contrast to reports from the same experimental group, concerning the nature of $\mathrm{UCo}_{2} \mathrm{Zn}_{20}$ and $\mathrm{URh}_{2} \mathrm{Zn}_{20}$. According to the alternative studies from another laboratory [1], the magnetic and thermodynamic data for $\mathrm{UIr}_{2} \mathrm{Zn}_{20}$ appear very similar to those reported for $\mathrm{UCo}_{2} \mathrm{Zn}_{20}$, and $\mathrm{UIr}_{2} \mathrm{Zn}_{20}$ is paramagnetic down to the lowest temperatures $(350 \mathrm{mK})$.

In our opinion, the presence of defects in some of the measured samples [3] may be one of possible reasons of the controversy between results of Refs. [1] and [2]. The basic electron characteristics, such as the electron density of states (DOS) or the shape of the Fermi surface may be influenced by the presence of the vacancy. In consequence, the relevant physical properties of the material under study can be changed. Here the positron annihilation spectroscopy is a one of most effective techniques to

*e-mail: A.Rubaszek@int.pan.wroc.pl probe the lattice perfection with respect to the presence of open volume defects [4].

In the present work we study sensitivity of the electron and positron density distributions and the positron lifetime to the presence of an $\mathrm{Ir}$ and $\mathrm{Zn}_{1}$ monovacancy in the $\mathrm{UIr}_{2} \mathrm{Zn}_{20}$ compound. Special attention is paid to the influence of the $\mathrm{e}-\mathrm{p}$ correlation effects on the resulting positron annihilation rate.

\section{Results and discussion}

The electron and positron band structure calculations have been performed for $\mathrm{UIr}_{2} \mathrm{Zn}_{20}$ using the linear muffin-tin orbital method (LMTO) in the atomic sphere approximation (ASA) [5] incorporating the local spin density approximation (LSDA) to the electronelectron exchange-correlation potential in the form proposed by Ceperley and Alder [6]. For the perfect material, the simple cubic supercell was used. It contained 184 atoms, which provided 2160 valence electrons (that occupy more than 1080 bands). The positions of $8 \mathrm{U}$, $16 \mathrm{Ir}, 16 \mathrm{Zn}_{1}, 48 \mathrm{Zn}_{2}$, and $96 \mathrm{Zn}_{3}$ atoms represent the $8 a$, $16 d, 16 c, 48 f$, and $96 g$ symmetry, respectively. This is an open structure with large interstitial space. In order to reduce the overlap of ASA, 136 empty spheres $\left(8 E_{1}\right.$, $32 E_{2}$, and $96 E_{3}$, which represent the interstitial region) have been added. The structural data (lattice constant $a$ and atomic positions) were taken from the experimental results, reported in [1]. The monovacancy was modelled by replacing the atomic sphere by the empty sphere of the same radii. The lattice constant $a$ remained unchanged [3].

As shown in Ref. [3], around the Fermi level the electron DOS spectrum exhibits a unique triple-peak structure, coming from uranium $5 f$ electrons, both for the perfect crystal as well as for the ones containing vacancy. Two sharp narrow peaks are due to $f_{5 / 2}$ and $f_{7 / 2}$ states. The remaining one may be interpreted in terms of the dual character of $5 f$ electrons in uranium [7]. 
The $\mathrm{Zn} 3 d$ electrons are localised in the energy range from about -0.55 Ry to -0.4 Ry below the Fermi level, as illustrated in Fig. 3 of Ref. [3]. The U $6 d$, Ir $5 d$, and U $5 f$ electrons are localised more close to the Fermi level. The presence of Ir vacancy shifts down the position of $\mathrm{Zn} d$ like bands with respect to the Fermi energy. As pointed out in Ref. [3], this tendency is also seen in the presence of $\mathrm{Zn}_{1}$ vacancy for the relevant Ir $5 d$ states. It is also apparent that Ir $5 d$ states are much less sensitive to Ir than to $\mathrm{Zn}_{1}$ vacancy. Finally, since the Ir $5 d^{7}$ states are found in the close vicinity of the Fermi level, the influence of the Ir vacancy on the resulting values of DOS is more pronounced in this energy region.

The electron density distribution in the individual atomic spheres, $n_{\mathrm{el}}(\boldsymbol{r})$, is drawn in Fig. 1. Results for a perfect crystal (stars) are compared with the ones for the Ir vacancy. The energy of $\mathrm{Zn} 3 d$ states is appreciably lower than the energy of its $5 d$ and $6 d$ counterparts from Ir and $\mathrm{U}$ atoms, respectively. In consequence, the Zn $3 d$ states are localised considerably deep inside the ASA sphere, as can be seen in the figure. The discrepancy between the distributions inside the particular $\mathrm{Zn}$ ASA is due to the difference in both the symmetry and nearest-neighbour atoms forming a cage-type structure $\left(\mathrm{Zn}_{3}\right.$ is located between the $\mathrm{U}$ and $\mathrm{Ir}$ atoms while $\mathrm{Zn}_{1}$ and $\mathrm{Zn}_{2}$ have $\mathrm{U}-\mathrm{U}$ and $\mathrm{Ir}-\mathrm{Ir}$ neighbours).

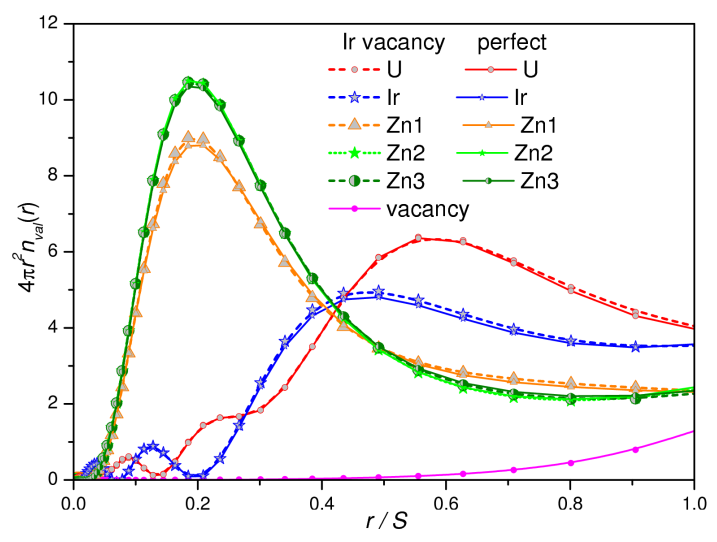

Fig. 1. The electron density distribution in the individual atomic spheres for a perfect crystal and the material containing an Ir vacancy.

It is apparent that $n_{\mathrm{el}}(\boldsymbol{r})$ is not very sensitive to the presence of the Ir vacancy. The same occurs for the $\mathrm{Zn}_{1}$ vacancy [3]. The removal of the $\mathrm{Ir}$ or $\mathrm{Zn}_{1}$ atom from the unit cell hardly causes the charge transfer between the individual atomic spheres. The electron charge contained inside the $\mathrm{Ir}$ and $\mathrm{Zn}_{1}$ vacancy spheres amounts to $0.58 \mathrm{e}$ and $0.21 e$, respectively, as specified in [3].

In contrast to electrons, the presence of vacancy considerably redistributes the positron density in the unit cell. This fact is illustrated in Fig. 2 for the case of the Ir vacancy. In the case of a perfect crystal, the U and Ir atomic spheres have a positive ionicity, and therefore the positron is repelled towards the $160 \mathrm{Zn}$ sites and

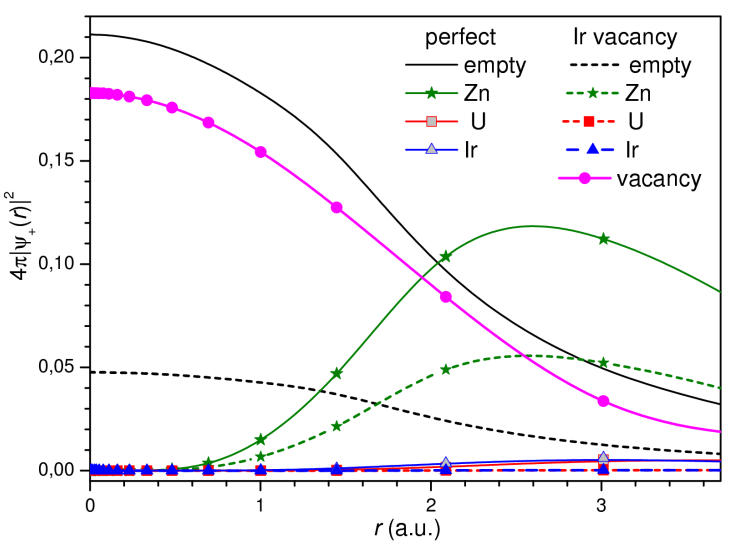

Fig. 2. Positron density distribution in the individual spheres. Solid lines represent the perfect crystal and the dashed lines correspond to the Ir vacancy.

the interstitial region, represented by 136 empty spheres. The single Ir vacancy sphere traps about $66 \%$ of positron charge. The considerable transfer of positron charge from both atomic and empty spheres towards the vacancy is well-observed. The relevant positron charge contained inside the individual atomic spheres is listed in Table 1 of Ref. [3]. The positron charge inside the single $\mathrm{Zn}_{1}$ vacancy amounts to about $57 \%$.

TABLE

Positron lifetime (in ps) in the perfect crystal and in the material containing monovacancy calculated within two various approaches to the positron screening by core electrons and two parameterisations of the $\mathrm{e}-\mathrm{p}$ correlation function for valence electrons. The bold numbers refer to the present state-selective approach with the GBN [3].

\begin{tabular}{c|c|c|c|c}
\hline \hline$\gamma[n]$ & Model & Perfect & Ir vacancy & Zn $_{1}$ vacancy \\
\hline Ref. [9] & ss & 147.6 & 229.8 & 251.6 \\
& sI & 132.2 & 214.7 & 239.6 \\
Ref. [10] & Ss & 154.7 & 237.7 & 260.4 \\
& sI & 146.8 & 228.1 & 250.2
\end{tabular}

The positron lifetime, $\tau$, is a unique characteristic of the material. This quantity is equal to the inverse of the total annihilation rate, $\lambda$, which is given by the formula

$$
1 / \tau=\lambda=\pi r_{0}^{2} c \sum_{S} \int_{\Omega} n_{\mathrm{el}}^{S}(\boldsymbol{r})\left|\psi_{+}(\boldsymbol{r})\right| \gamma(S, \boldsymbol{r}) \mathrm{d} \boldsymbol{r},
$$

where $r_{0}$ and $c$ denote the classical electron radius and velocity of light, respectively, and $\Omega$ is the volume of the sample. Index $S$ in (1) stands for the type of electrons, core and valence, and $n_{\mathrm{el}}^{S}(\boldsymbol{r})$ is the relevant electron charge density in the host material. The parameter $\gamma$ denotes the electron-positron $(\mathrm{e}-\mathrm{p})$ correlation function that depends on both the type of electrons (core, valence), $S$, and the positron position, $\boldsymbol{r}$ [8].

Let us pay more attention to the e-p correlation function. In the literature, this quantity has been most commonly approximated within the LDA as a function of the 
local electron density on the positron position, $\gamma\left[n_{\mathrm{el}}(\boldsymbol{r})\right]$ (see [8] and references cited therein). Here the Boroński and Nieminen [9] (GBN) parameterisation gas is most popular. However, when the state-independent (sI) approach has been applied to core electrons (i.e. the correlation function in Eq. (1) was common for valence and core electrons), the resulting annihilation rates have overestimated the experimental values in a series of metals [8], especially for these elements in which core electrons are spread inside the Wigner-Seitz cell. Application of the state-selective (ss) approximation, which utilises the energy-dependent enhancement factors, with energy $E=0$ for core electrons [8], improved agreement between theory and experiment. Other methods of increasing the values of $\tau$ consist in going beyond the LDA within the ss or sI approaches. Recently Drummond et al. [10] performed alternative calculations of the e- $\mathrm{p}$ correlation function in an electron gas (GDR), using the Monte Carlo simulation. The resulting GDR has been parameterised as a function of the electron density parameter, $r_{\mathrm{s}}=[3 / 4 \pi n]^{1 / 3}$. The values of GDR are continuously lower than GBN in the wide range of electron densities. The differences increase with increasing $r_{\mathrm{s}}$. Surprisingly, the GDR contact density exceeds the low density limit $\left(r_{\mathrm{s}} \rightarrow \infty\right)$ equal to $1 / 8 \pi$. It is obvious that incorporating the GDR in Eq. (1) leads to increase of the positron lifetime as compared to GBN.

Calculations of the present work have been performed within the ss LDA with the GBN, following Ref. [8]. Results are listed in the first row of Table (bold numbers) and they are compared with the positron lifetime obtained for GDR within ss and sI models as well as for GBN and sI.

The value of the positron lifetime calculated for a perfect crystal is compared with its counterpart for the Ir and $\mathrm{Zn}_{1}$ vacancy in Table. The first and second row represent the results obtained, respectively, for the GBN [9] with ss (bold numbers) and sI models. The third and fourth rows correspond to the Drummond et al. [10] parameterisation of $\gamma\left[n_{\mathrm{el}}(\boldsymbol{r})\right]$.

Positron trapping inside the vacancy is clearly reflected in the positron lifetime values, as specified in the table. The positron lifetime in a perfect crystal, calculated according to (1) within the state selective approach to the $\mathrm{e}-\mathrm{p}$ correlation function and GBN, amounts to $147.6 \mathrm{ps}$. The present result for a positron lifetime in the perfect $\mathrm{UIr}_{2} \mathrm{Zn}_{20}$ is very close to the experimental value for a perfect Zn, that amounts to 146 ps [11]. This feature is in agreement with the expectations, as $\mathrm{UIr}_{2} \mathrm{Zn}_{20}$ is a zinc-based material with small contamination from $\mathrm{U}$ and Ir atoms. Therefore, the comparison of the theoretical value of $\tau$ for the perfect $\mathrm{UIr}_{2} \mathrm{Zn}_{20}$ with the relevant experimental data for $\mathrm{Zn}$ seems to justify the present calculation. It should be noted here that the sI model with the GDR leads to a quite similar result.

The positron lifetime is considerably longer in the crystal containing a monovacancy than in a perfect material. It amounts to 229.8 and $251.6 \mathrm{ps}$ for the $\mathrm{Ir}$ and $\mathrm{Zn}_{1}$ va- cancy, respectively. The important thing to note when comparing values of $\tau$ in the perfect crystal and in the material containing monovacancy, is that the positron lifetime in the $\mathrm{UIr}_{2} \mathrm{Zn}_{20}$ compound is very sensitive to the presence and type of vacancy. Therefore, the measurements of the lifetime spectrum in this material would be highly required in order to probe the lattice imperfections in the samples under study. In particular, the knowledge of the positron annihilation characteristics in $\mathrm{UIr}_{2} \mathrm{Zn}_{20}$ crystals would be very helpful in the interpretation of the basic physical properties of the crystals investigated in [1] and [2].

Finally, let us pay some attention to the influence of the approach to the $\mathrm{e}-\mathrm{p}$ interaction on the lifetime value calculated according to formula (1). Since $\left.\left.\gamma^{\mathrm{LDA}}\right] 0, n_{\mathrm{el}}(\boldsymbol{r})\right] \ll$ $\left.\left.\gamma^{\mathrm{LDA}}\right] n_{\mathrm{el}}(\boldsymbol{r})\right]$, the relevant value of $\lambda$ obtained within the sI approach is also greater than its ss counterpart. The discrepancy between these approaches indicates that the core electron contribution to the positron annihilation rate (1) is not negligible. Moreover, the GDR $\ll$ GBN and, in consequence, the effect of incorporating the state-selectivity in Eq. (1) is similar to applying GDR instead of GBN within the sI model.

\section{Conclusions}

The value of the positron lifetime is quite sensitive to the approach to the electron-positron correlation function. The presence of monovacancy strongly influences the positron distribution in the unit cell and the resulting positron annihilation characteristic, $\tau$. Moreover, positron lifetime appears to be sensitive to the type of vacancy in this compound. Therefore, the experimental lifetime spectra would provide reliable information on the possible defects in the samples. For this reason, carrying out relevant measurements would be highly recommended.

\section{References}

[1] P. Swatek, M. Daszkiewicz, D. Kaczorowski, Phys. Rev. B 85, 094426 (2012).

[2] E.D. Bauer, A.D. Christianson, J.S. Gardner, V.A. Sidorov, J.D. Thompson, J.L. Sarrao, M.F. Hundley, Phys. Rev. B 74, 155118 (2006).

[3] A. Rubaszek, Phys. Status Solidi B 250, 1404 (2013).

[4] For review and references see e.g. M.J. Puska, R.M. Nieminen, Rev. Mod. Phys. 66, 841 (1994); R.M. Nieminen, Positron Spectroscopy of Solids, Eds. A. Dupasquier, A.P. Mills, IOS Press, Amsterdam 1996, p. 443; O. Melikhova, J. Cizek, J. Kuriplach, I. Prochazka, M. Cieslar, W. Anwand, G. Brauer, Intermetallics 18, 592 (2010); G. Brauer, W. Anwand, D. Grambole, J. Grenzer, W. Skorupa, J. Cizek, J. Kuriplach, I. Prochazka, C.C. Ling, C.K. So, D. Schulz, D. Klimm, Phys. Rev. B 79, 115212 (2009). 
[5] O.K. Andersen, Phys. Rev. B 12, 3060 (1975); W.R.L. Lambrecht, O.K. Andersen, Phys. Rev. B $\mathbf{3 4}$, 2439 (1986); H.L. Skriver, in: The LMTO Method. Muffin-Tin Orbitals and Electronic Structure, Eds. M. Cardona, P. Fulde, Springer-Verlag, Berlin 1984.

[6] D.M. Ceperley, B.J. Alder, Phys. Rev. Lett. 45, 566 (1980); A.D. Becke, Phys. Rev. A 38, 3098 (1988); J.P. Perdew, Y. Wang, Phys. Rev. B 45, 13244 (1992).

[7] G. Zwicknagl, P. Fulde, J. Phys. Condens. Matter 15, S1911 (2003).
[8] S. Daniuk, M. Sob, A. Rubaszek, Phys. Rev. B 43, 2580 (1991)

[9] E. Boroński, R.M. Nieminen, Phys. Rev. B 34, 3820 (1986).

[10] N.D. Drummond, P. Lopez Rios, R.J. Needs, C.J. Pickard, Phys. Rev. Lett. 107, 207402 (2011).

[11] P. Seeger, F. Banhart, W. Brauer, in: Positron Annihilation, Eds. L. Dorikens-Vanpraet, M. Dorikens, P. Seeger, World Sci., Singapore 1989, p. 275. 